\title{
The Simulation Experiments on Impulse Characteristics of Tower Grounding Devices in Layered Soil
}

\author{
Leishi Xiao, Qian Li, Zhangquan Rao, Wenjun Zhou, and Jiarui Huang
}

\begin{abstract}
Reducing the impulse grounding resistance of transmission line tower grounding system is the major measure to improve the lightning impulse withstand level and to ensure power system stability. For tower mainly adopts box with radiation grounding devices, this paper uses simulation experiments method based on similarity theory, researches the influence of radiation length, radiation angle and the proportion of box and radiation, branch length on the box with radiation grounding devices' impulse characteristics in layer soil model. Results of the experiments show that the branches have an effective use of length, the box and radiation have an optimal proportion, over length radiation or improper proportion will render the resistance reduction ineffective. According to the results of the experiments, we have gotten the optimal length rays add branch and put forward an impulse characteristics features better tower grounding structure which is named T2-15-25\&H2-15. There are some references for engineering.
\end{abstract}

Index Terms - Tower grounding system, impulse grounding resistance, ground network structure, impulse characteristics, simulation experiments, resistance reduction.

\section{INTRODUCTION}

Reducing the impulse grounding resistance of transmission line tower grounding system is the effective measure to improve the lightning impulse withstand level [1], the impulse grounding resistance will directly affect the line lighting protection effect [2]. Lighting current flows into the earth through the grounding conductor, it will increase the local field strength around the conductor and make the spark discharge happened when the field strength exceeds the soil breakdown electric field strength [3]. And the spark discharge will increase the equivalent grounding radius and the soil conductivity [3]. At the same time, lighting electric wave frequency component are mainly concentrated in $10 \sim 200 \mathrm{kHz}$, the conductor inductance and the inductance to the ground will prevent the current from flowing to the distant grounding part. Due to the inductive effect, the conductor will not be used sufficiently [4]. The spark effect and the inductive effect will make a large difference between the power frequency grounding resistance and the impulse grounding resistance. The method to reduce the power frequency grounding resistance is not applicable to the impulse grounding resistance.

Manuscript received September 14, 2015; revised December 12, 2015.

Leishi Xiao, Qian Li, and Zhangquan Rao are with the Guangdong Electric Power Research Institute of China Southern Power Grid, China (e-mail: xiaoleishi@126.com,gdliqian@126.com,30069274@qq.com).

Wenjun Zhou and Jiarui Huang are with Country College of electrical engineering in Wuhan University, China (e-mail: wjzhou@whu.edu.cn, hjr947@foxmail.com).
The existing resistance reduction methods mainly include using the resistance reducing agent, replacing the local soil around the grounding conductor, changing the grounding network structure. The resistance reducing agent can improve the electrical conductivity of the soil around the grounding electrodes directly. But its low adhesion, easy solubility, corrosion to the conductor and the pollution to the soil have limited its application [5]. There has been happened to use the replacement of soil to reduce the grounding resistance in engineering [6]-[8], namely to replace the soil of high resistivity around the grounding conductor by the soil of low resistivity. The literature [9] has established a "replacement of soil" model to calculate the grounding resistance, and it indicates that the resistance reduction rate is lower than $5 \%$. The change of grounding structure mainly includes extending the radiation length, adding some branches on the radiation and so on. For the high resistivity area, to increase the radiation length can effectively reduce the power frequency grounding resistance, but the resistance reduction effect is not obvious to the impulse grounding resistance. In some special regions, the radiation length has been more than $200 \mathrm{~m}$ and the power frequency grounding resistance is less than $2 \Omega$, but the accident of lighting flashover to cause the tripping operation is still happened [10]. Exceeding the electrode is not equivalent to reducing the impulse grounding resistance. There are also many applications of adding conductors on the radiation branch. The literature [11], [12] has studied the way to reduce the impulse resistance by adding the branch in the perspective of improving the flowing current distribution. But they do not take the size of conductors into account and have not gotten the optimal length of the radiation and the branch of the tower grounding system directly.

In order to optimize the impulse characteristics of the grounding network in two-layer soil [13] and reduce the impulse grounding resistance effectively, this paper adopts the method of simulation test [14] and test the usual box with radiation grounding system used in the transmission line tower. The influences of the radiation length, radiation angle, the proportion of box and radiation length to the impulse properties will be studied. At the same time, this paper researches the influence of branch length to the horizontal grounding conductor and the box grounding conductor in the condition of a total length. By comparing the impulse features of different structure of grounding systems, an optimized arrangement of grounding network has been proposed.

\section{EXPERIMENTAL METHOD}

The testing place is the grounding laboratory located in Chinese Electric Power Research Institute UHV AC test site. 
The experimental wiring arrangement is shown in Fig. 1. The grounding conductor is buried in a hemispherical simulation slot (Fig. 2) whose diameter is $8 \mathrm{~m}$. The slot is filled with soil and the slot wall is used for reflowing. The testing power is an impulse current generator which can produce the $60 \mathrm{kV} / 10 \mathrm{kA}$ current. Through changing the adjustable resistance and inductance, the needed current is exported and injected into the grounding device. The current flows through the soil and is collected by the slot wall, then back into the low voltage terminal of the impulse current generator. The testing voltage divider is the weak damping resistor voltage divider whose voltage division ratio is $115.7: 1$, used for measuring the voltage waveform in the feed point. The shunt is the Pearson coil whose changing ratio is $0.01 \mathrm{~V} / \mathrm{A}$, used for measuring the loop current waveform. The soil resistivity in the hemispherical slot is $30 \Omega \cdot \mathrm{m}$. $35 \mathrm{~cm}$ thick silver sand whose resistivity is $2180 \Omega \cdot \mathrm{m}$ has been laid on the soil, so a double-layer soil model is gotten. To simulate the impulse features in high soil resistivity area, the simulated grounding electrode is buried in the sand.

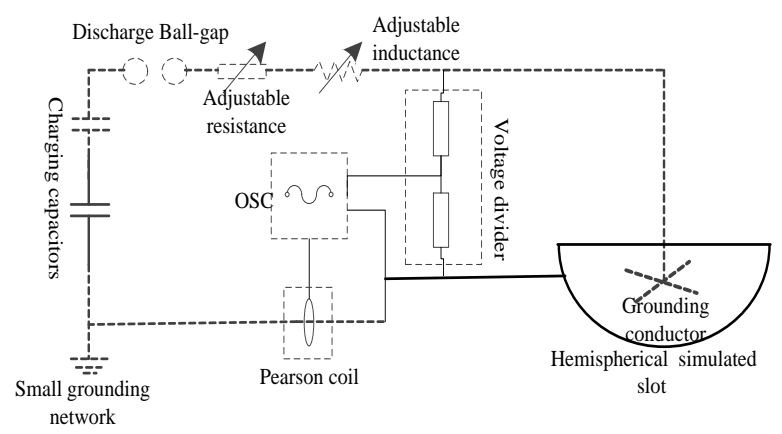

Fig. 1. Connecting diagram of experiment.

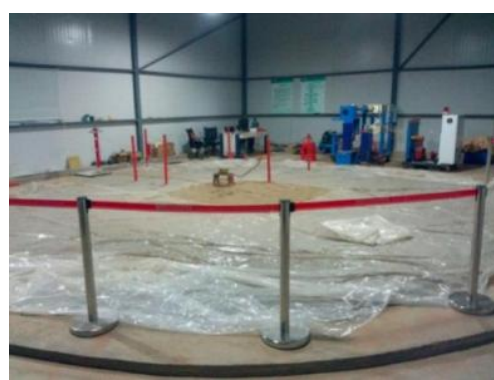

Fig. 2. Photo of the experiments site.

According to similarity theory, the paper takes the main simulation scale [15] as follows:

Soil resistivity: $\rho_{1}=\rho_{2}$

Soil breakdown electric field strength: $E_{01}=E_{02}$

Length: $l_{1}=n l_{2}$

Time: $\quad t_{1}=n t_{2}$

Amplitude of impulse current: $I_{\mathrm{m} 1}=n^{2} I_{m 2}$

Wave front time of impulse current: $\tau_{1}=n \tau_{2}$ Impulse grounding resistance: $R_{1}=R_{2} / n$

Impulse coefficient: $\alpha_{1}=\alpha_{2}$

In each equation, the physical quantity in the subscript 1 represents the true type of grounding device and the one in the subscript 2 represents the simulated type of grounding device. And the analog scale is $\mathrm{n}$. The wave front time of impulse current will greatly influence the impulse characteristic of grounding device. In order to make the wave front time of testing impulse current close to the standard lighting current and reduce the loop inductance, the current will be flowed into the earth by three copper braided straps. Because of the size of simulation test electrode shall not exceed 1/4 times the diameter of the simulated slot [16], considering to the test requirement for wave front time and the size of electrode, the simulation ratio has been set as $n=50: 1$. The wave front time of impulse current in the test is between $0.08 \mu \mathrm{s} \sim 0.14 \mu \mathrm{s}$, as it is shown in Fig. 3. After the conversion, the wave front time is between $4 \mu \mathrm{s} \sim 7 \mu \mathrm{s}$. It is relatively close to the wave front time of standard lighting current which is $1 \sim 4 \mu \mathrm{s}$. In the real tower grounding device whose structure is the box with radiation, the length of the box side is commonly $8 \mathrm{~m} \sim 15 \mathrm{~m}$, the radiation length is 0 60m. The shapes and sizes of grounding device are shown in Table I, the influence of radiation length, radiation angle and the proportion of box and radiation, branch length on the box with radiation grounding devices' impulse characteristics in layer soil model have been discussed.

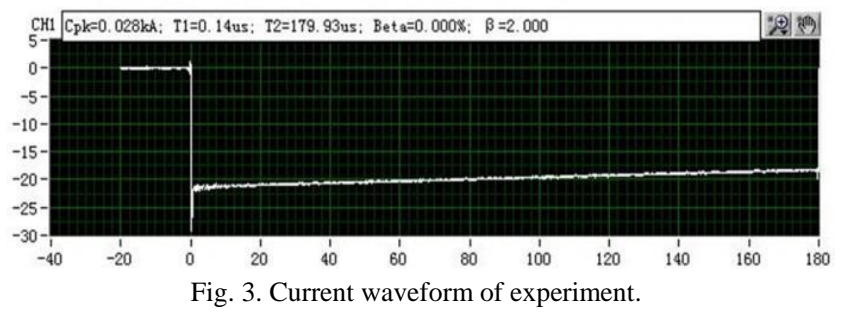

TABLE I: STRUCTURE OF THE EXPERIMENTS GROUNDING DEVICES

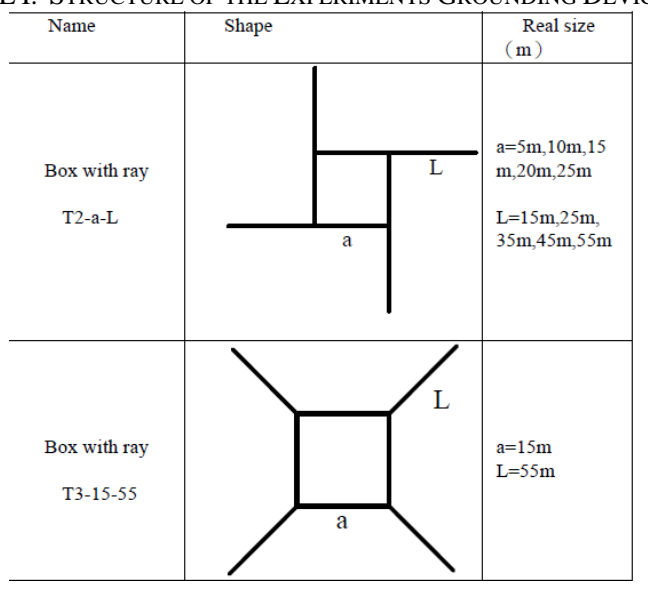

\section{ANALYSIS OF TEST RESULTS}

\section{A. Influence of Radiation Length}

In the high soil resistivity area, in order to reduce the grounding resistance, the radiation length is more than $60 \mathrm{~m}$ generally. But as the radiation length had increased, the inductive effect of grounding device also increased. It makes the effective utilization rate of grounding device reduced. When the length reaches a certain value, the impulse grounding resistance will increase slowly. To explore the effective length of the radiation length in the box with radiation grounding device under the condition of high soil 
resistivity, the paper will study the impulse characteristics of the condition that the radiation length is $15 \mathrm{~m}, 25 \mathrm{~m}, 35 \mathrm{~m}, 45 \mathrm{~m}, 55 \mathrm{~m}$ with the length of the box side is $15 \mathrm{~m}$ respectively. ZC-8 type grounding resistance shaking table is used to measure the power frequency grounding resistance of the above five kinds of grounding devices. The grounding resistances transformed by the simulation result are shown in Table II.

The impulse characteristic of different grounding devices are shown in Fig. 4. The amplitude of impulse current injected into the grounding device is very high. It makes the effect of spark is stronger than the inductive effect, so the impulse coefficient are less than 1 . The test results show that with the increase of current amplitude, the impulse grounding resistances of different grounding devices are reduced. Due to the increase of impulse current amplitude, the partial field strength around the grounding device will increase. When the value is higher than the soil breakdown electric field strength, the spark discharge phenomenon will appear. Ignoring the residual resistivity of the spark discharge area and assuming that the potential of the partial discharge area is the same to the potential of grounding device, it is equivalent to the condition of increasing the diameter of grounding device and expanding the area of current flow. And it will reduce the impulse grounding resistance. But if the amplitude increases to a certain degree, the spark discharge tends to be saturated. It makes that the falling trend of the impulse grounding resistance is more and more unobvious. In the condition of the same current amplitude, Fig. 5 shows the variation tendency of the grounding device with different radiation length. When the length of the ray reaches $55 \mathrm{~m}$, the inductive effect will be more obvious and the impulse grounding resistance will not decrease at all. All of this tells us, an effective length of the radiation length is existed. It is not useful to set a very long radiation length.

TABLE II: POWER FREQUENCY GROUNDING RESISTANCE OF DIFFERENT GROUNDING ELECTRODES

\begin{tabular}{|c|l|l|l|l|c|}
\hline Code & $\begin{array}{l}\text { T2-1 } \\
5-15\end{array}$ & $\begin{array}{l}\text { T2-15- } \\
25\end{array}$ & $\begin{array}{l}\text { T2-15- } \\
35\end{array}$ & $\begin{array}{l}\text { T2-15- } \\
45\end{array}$ & $\begin{array}{l}\text { T2-15- } \\
55\end{array}$ \\
\hline $\begin{array}{l}\text { Power } \\
\text { frequency } \\
\text { grounding } \\
\text { resistance } \\
(\Omega)\end{array}$ & 23.9 & 17.5 & 13.6 & 11.1 & 9.4 \\
\hline
\end{tabular}

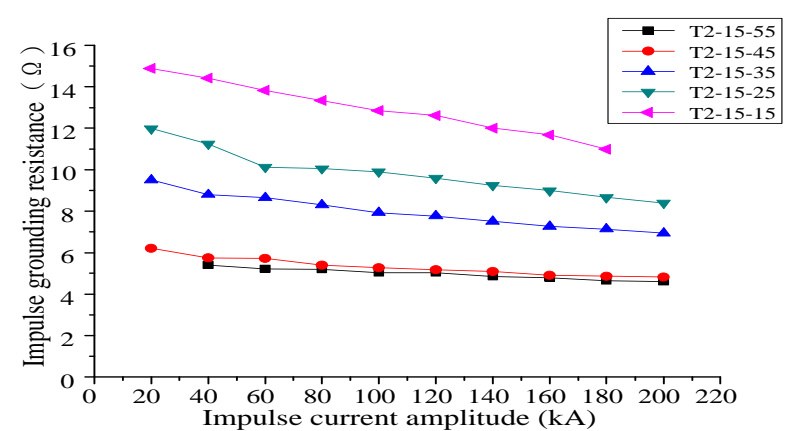

Fig. 4. Impulse characteristics of box grounding devices in different radiation length.

\section{B. Influence of Ray Angle}

To study the effect of ray angle, this paper had compared the impulse characteristic of T2-15-55 and T3-15-55 in Tab. I.
The two kinds of grounding devices have the same length of box side and radiation length. The test result is shown in Fig. 6. They are almost the same. So the ray angle has a little influence when the radiation length is comparatively long.

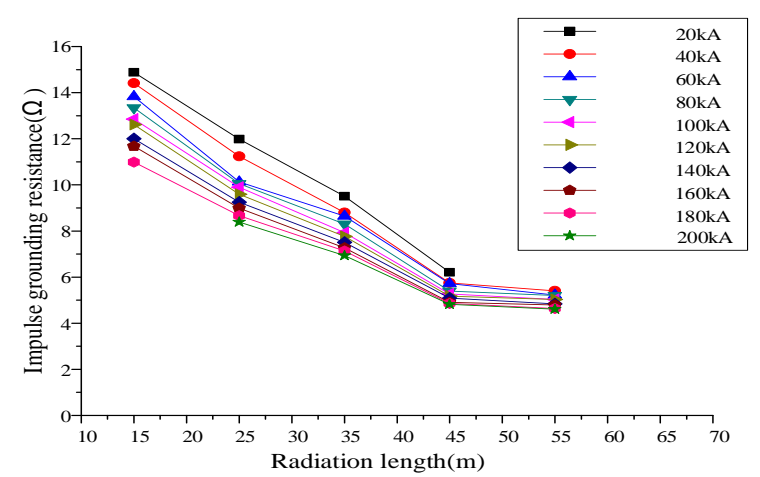

Fig. 5. Effects of the length of radiation on the impulse grounding resistance.

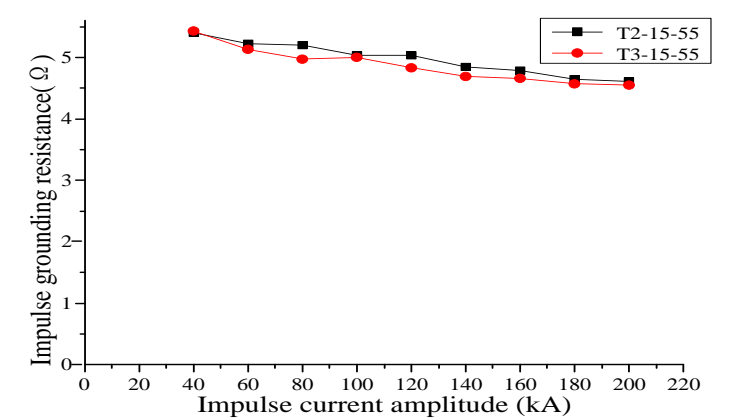

Fig. 6. Impulse characteristics of box grounding devices in different radiation angle.

\section{Influence of the Proportion of Box and Radiation}

When the total length is a certain value, how to get the optimal effect by controlling the box length and the radiation length? The paper had tested the five kinds of box with radiation grounding device: $\mathrm{T} 2-5-65$, T2-10-60, T2-15-55, T2-20-50, T2-25-45. The total lengths of these grounding devices are $70 \mathrm{~m}$. The proportion of box and radiation are respectively $1 / 13,1 / 6,3 / 11,2 / 5,5 / 9$, the results are shown in Fig. 7. As the proportion increases from $1 / 13$ to $5 / 9$, the impulse grounding resistance decreases firstly and then increases. With the increase of the length of box side, the conductor spacing increases. It makes the mutual impedance decrease and the grounding resistance decrease. But the field strength generated by the impulse current also decreases, the spark effect weakens and the grounding resistance increases. When the proportion is less than $1 / 6$, the mutual impedance plays a leading role; when the proportion is greater than $3 / 11$, the spark effect plays a leading role. So it is appropriate to set the proportion between 1/6 3/11.

\section{Influence of Branch Length on the Radiation}

In the actual grounding transformation, to add the branch on the radiation is often used to reduce the impulse grounding resistance. So the paper researches its rules. The result of horizontal grounding device is shown in Fig. 8. When the total length is a certain value $(60 \mathrm{~m})$, adding the branch can reduce the impulse grounding resistance of horizontal grounding device. With the increase of the branch, the 
impulse grounding resistance decreases firstly and increases then. It is similar to the proportion of box and radiation. The appropriate proportion of branch and radiation need to be explored.

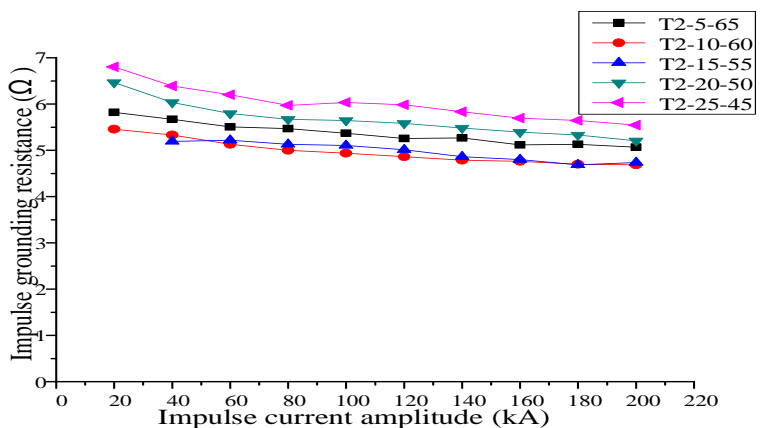

Fig. 8. Impulse characteristics of grounding devices in different proportion of box side and radiation length.

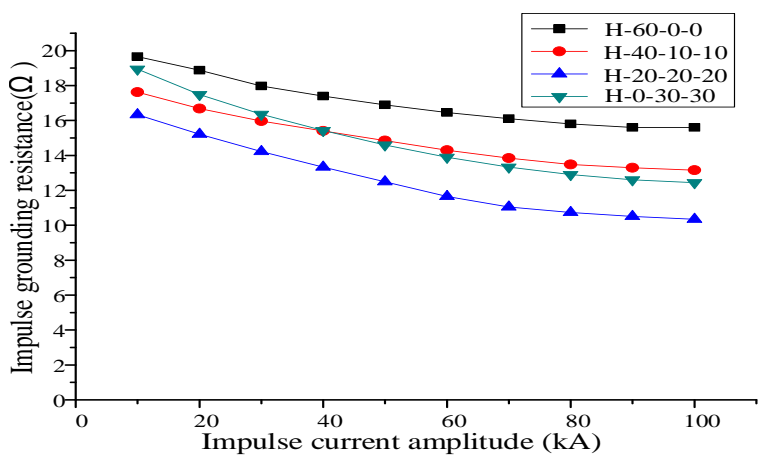

Fig. 7. The impulse characteristics of horizontal grounding devices in different branch length.

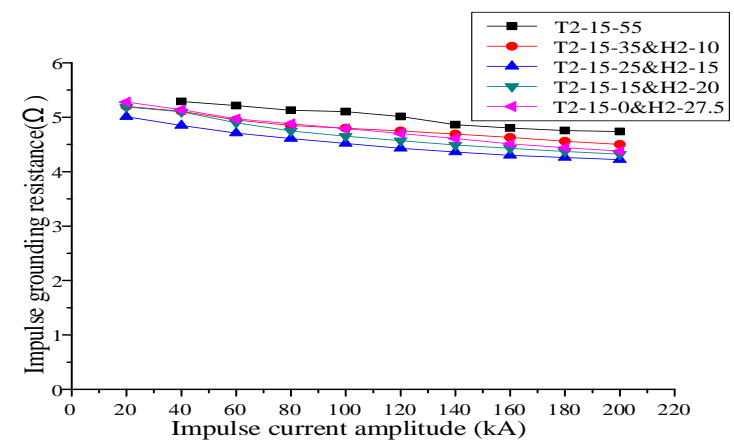

Fig. 9. The impulse characteristics of box grounding devices in different branch length.

In order to explore the influence of the branch length on the radiation on the impulse characteristic, the paper selects the five kinds of grounding devices: T2-15-55, T2-15-35\&H2-10, T2-15-25\&H2-15, T2-15-15\&H2-20 and T2-15-0\&H27.5 in Tab. I (d). In these grounding devices, the length of the box side is a, equal to $15 \mathrm{~m}$, the total length of radiations and branches is $55 \mathrm{~m}$, the lengths of branches $\mathrm{L} 1$ are $0 \mathrm{~m}, 10 \mathrm{~m}, 15 \mathrm{~m}, 20 \mathrm{~m}, 27.5 \mathrm{~m}$. The special one $\mathrm{T} 2-15-0 \& \mathrm{H} 27.5$ represents that the radiation length is $0 \mathrm{~m}$ and the branches are connected to the four vertices of the grounding system. The test result is shown in Fig. 9. It is Similar to the horizontal grounding electrode. In the same condition, with the increasing of branch length, the impulse grounding resistance decreases firstly and increases then. Among these grounding devices, the T2-15-25\&H2-15 one is the best. To a certain extent, the branches can reduce the impulse grounding resistance. But on the opposite, the branches will also influence the efficiency that the current flows into the earth. So it is necessary to choose the optimal branch length on the radiation.

\section{CONCLUSIONS}

In the two-layer soil model, the method of simulation test is been used to study the impulse characteristic of the box with radiations grounding devices used by the transmission line tower grounding devices. And the influences to the impulse characteristic, including the radiation length, the radiation angle, the proportion of box and radiation and the branch, are been tested.

The radiation angle has little influence on the impulse characteristic. There is a certain value for a special radiation line, once exceeding the value, the impulse grounding resistance decreases slowly. There is an optimal proportion of box and radiation to get the best grounding resistance reducing effect. All of this could be the guidance in the design or transformation of the grounding system.

There will be some effect to add the branch on the radiation. When the total length of the radiations and branches is a certain value, there will be an optimal branch length for the best resistance reducing effect. The simulation test shows that in the two-layer soil model(the upper soil resistivity is about $2180 \Omega \cdot \mathrm{m}$, the lower soil resistivity is about $30 \Omega \cdot \mathrm{m})$, the $\mathrm{T} 2-15-25 \& \mathrm{H} 2-15$ kind grounding structure has the minimum impulse grounding resistance among all of the tested grounding devices. The structure and the proportion can offer some references for engineering.

\section{REFERENCES}

[1] G. Z. Guan, High Voltage Engineering Fundamentals, 1st ed. Beijing, China: China Electric Power Press, 2003, pp. 154-155.

[2] M. E. Almeida and M. T. C. D. Barros, "Accurate modeling of rod driven tower footing," IEEE Trans. Power Delivery, vol. 11, pp. 1606-1609, 1996.

[3] P. L. Bellaschi, "Impulse and 60-cycle characteristics of driven ground-II," Amer. Inst. Elect. Eng. Trans, vol. 61, pp. 349-363, 1942.

[4] N. M. Nor, S. Srisakot, H. Griffiths, and Haddad, "Characterization of soil ionization under fast impulses," in Proc. 25th International Conference on Lightning Protection, 2000, pp. 417-422A.

[5] J. Liu, L. Y. Ye, X. P. Zhang et al., "Research on heavy current impulse characteristics of long-acting chemical resistance reducer grounding electrode," High Voltage Engineering, vol. 3, pp.1-8, 1981.

[6] S. Y. Tang, W. Q. Mo, and L. Y. Zhou, "Grounding treatment in substation in high soil resistivity area," High Voltage Engineering, vol. 32, pp. 121-122, 2006.

[7] J. L. Li, R. C. Zheng, T. F. Yang, et al, "Discussion on defining the values of related parameters about grounding project," High Vohage Apparatus, vol. 40, pp. 264-266, 2004.

[8] T. Z, Yao and J. F. Wang, "Grounding resistance calculation of soil-exchanging method based on resistance reduction technology of backfilled soil," Guangxi Electric Power, vol. 3, pp. 81-82, 2007.

[9] Y. G. Liu, X. E. Liu, X. D. Yu, L. S. Xiao et al., "Calculation and analysis of grounding parameters of grounding grid with artificial improvement soil surrounding the grounding grid's conductors," High Voltage Apparatus, vol.46, pp. 16-19, 2010.

[10] A. C. Liew and M. Darveniza, "Dynamic model of impulse characteristics of concentrated earths," in Proc. the Institution of Electrical Engineers, 1974, vol. 121, pp.123-135.

[11] W. X. Sima, C. P. Lei, T. Yuan et al., "Experimental study on grounding resistance reduction based on improved grounding electric field distribution induced by the diffuser of impulse current," High Voltage Engineering, vol. 37, pp. 2294-2301, 2011. 
[12] T. Yuan, C. P. Lei, W. X. SIMA et al., "Analysis of grounding resistance reduction effect based on enhancing impulse current leakage efficiency," Transactions of China Electrotechnical Society, vol. 27, pp. 276-284, 2012.

[13] G. Yu, W. M. Sun, and J. L. He, "Optimal design rules of grounding system considering seasonal influence," High Voltage Apparatus, vol. 41, pp. 104-106, 2005

[14] J. L. He, R. Zeng, Y. P. Tu et al., "Laboratory investigation of impulse characteristics of transmission tower grounding devices," IEEE Transaction on Power Delivery, vol. 18, pp. 994-1000, 2003.

[15] Y. Q. Gao, "Research on soil breakdown mechanism and grounding system transient characteristics," Ph.D. dissertation, Beijing: Tsinghua University, 2003.

[16] J. L. He and X. L. Chen, "Simulation experiments theory of the impulse resistance characteristics of transmission line ground devices," Journal of Tsinghua University (Science and Technology, vol. 34, pp. 38-43, 1994.

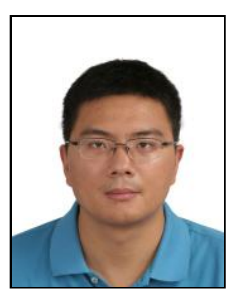

Leishi Xiao was born in China in 1987. He received the M.S. degree in 2010 from Chongqing University of Electrical Engineering. Currently. He is working in Guangdong Electric Power Research Institute of China Southern Power Grid since 2010. He is mainly engaged in electric power system grounding overvoltage and research.

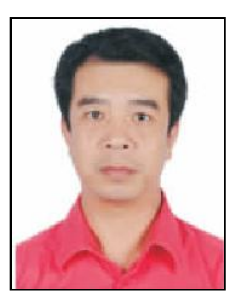

Qian Li was born in Guangxi province, China in 1965 He received the Ph.D. degree in 1996 from School of Electrical and Electronic Engineering HUST. He is mainly engaged in overvoltage and insulation coordination, electric power system grounding. He works at Guangdong Electric Power Research Institute, Guangzhou, China .

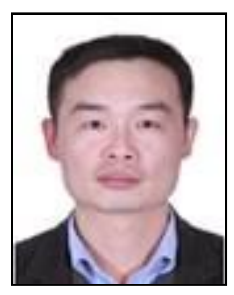

Zhangquan Rao was born in Hubei province, China in 1980. He received the master degree in electrical engineering from Wuhan University, Wuhan, China in 2005. He has worked at Guangdong Electric Power Research Institute, Guangzhou, China since 2005. His major research fields are high voltage insulation and high voltage test techniques.

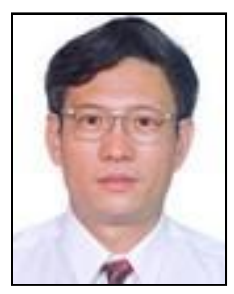

Wenjun Zhou was born in China in 1959. He received the Ph.D. degree in 1990 from Wuhan University of Hydraulic and Electrical Engineering. Currently, he is a professor at the School of Electrical Engineering of Wuhan University, China. He is a member of the High Voltage Committee of the Chinese Society of Electrical Engineering (CSEE), the Electro-technical Test and Measurement Committee of the China Electro-technical Society (CES), and the China Lightning Protection Standard Committee. He is also the vice director of the Hubei High Voltage Committee. His research interests include lightning protection and the diagnostic techniques for outdoor electrical insulations.

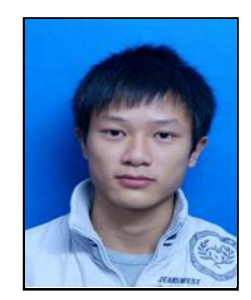

Jiarui Huang was born in China in 1994. He has been studying in Wuhan University since 2014.

His major research field is electric power system grounding. 